\title{
Research on Dynamic Visualization Teaching Method of
}

\section{Computer Network}

\author{
Yang Fan $^{1}$, Zhao Li-zhen ${ }^{2}$ \\ ${ }^{1}$ School of Computer Science, Zhaoqing University, Zhaoqing, Guangdong, China . \\ ${ }^{2}$ Educational Technology and Computer Center Zhaoqing University, Zhaoqing, Guangdong, \\ China
}

Keywords: Computer network, Dynamic, Visualization, Teaching methods, Virtual network

\begin{abstract}
According to the problems of Computer Network course, namely,knowledge is abstract, the teaching materials are complicated and ambiguous, the development of technology changes quickly, the learning burden is large, etc.. It introduced a virtual network teaching environment and mind map, so as to show the main network technologies and teaching resources of protocols by using virtual network environment and network typical cases, by using mind map to make teaching courseware in line with associative thinking process of the students. During the period of explaining concepts and protocols in classroom, it can use dynamic operation of the network teaching resources and network cases instead of the static network topology diagram, through Console, Wireshark, SocketTool and other tools, it can check network equipment and link information, subnet information and identification, network routing, network protocol, the state of network service, TCP/UDP port, as well as the contents of the packet of the specific data. Thus it can show the operation of network by means of dynamic interaction and visualization, so as to form a dynamic visual teaching method.
\end{abstract}

\section{Introduction}

The main problems of Computer Network course teaching are as follows:the first one is curriculum content, which is broad with various concept of knowledge, the control principle of protocol is abstract, which is difficult to use natural language linear to have clear expression for the network communication process ${ }^{[1]}$; the second problem is the teaching content is concept biased and the protocol described may weaken the practical control ability of the computer network itself; the third problem is the content of textbook that may be correct, but it still is lack of integrity and accuracy $\left.{ }^{[2][3}\right]$, therefore, it is difficult for students to fully understand the correct knowledge; even they understand the single point of knowledge, students are also very difficult to quickly establish the relationship among the knowledge points, it is difficult to identify knowledge in real network status and function, which can result in the loss of students' interest in learning; the forth problem is the technical development, which is changing so quickly that the learning load of students is great.

The proposed dynamic visualization teaching method in this paper is based on fully understanding teaching content and teaching target of the curriculum, by the means of virtual network environment and network teaching, combined with the knowledge map and mind 
map with courseware, using content related concepts, associative performance visualization method of computer network protocol and technology. Moreover, can use dynamic operation of the network teaching resources and network cases instead of the static network topology diagram, through Console, Wireshark, SocketTool and other tools, it can check network equipment and link information, subnet information and identification, network routing, network protocol, the state of network service, TCP/UDP port, as well as the contents of the packet of the specific data. It also can use dynamic interactive method directly and accurately to display and analyze the operation of network operation, as well as the real operation of computer network itself, which can help students quickly understand concept, and master the relevant technical principles effectively.

\section{Dynamic Visualization Technology Elements Analysis}

\section{Virtual Network Environment}

The virtual network environment ${ }^{[4]}$ consists of network simulator and virtual machine, the network simulator can be eNSP or GNS3. The virtual network environment can be used to build a virtual network case for real network applications, which can provide network capabilities research, teaching demonstration, network test method training and so on.

eNSP is a network simulator based on Huawei network device ${ }^{[5]}$, which can provide the equipment connection, equipment configuration and network function simulation ability for Huawei network. GNS3 is a network simulator based on Cisco network device ${ }^{[6]}$, which can provide the equipment configuration and function simulation ability of Cisco network. VirtualBox is a free virtual machine platform published by Oracle company ${ }^{[7]}$, a series of 32/64 virtualization virtual machine can mainly have three functions in the virtual network environment, the first is to provide the running environment of the virtual network devices, followed by the operating environment provides complex network application service, the third is to simulate real network client. From the capabilities of networking, VirtualBOX virtual machine can provide six different networking patterns ${ }^{[8]}$, which can enable multiple connections with GNS3 network simulator and real network.

\section{Analysis on Data Packet Capture Technology}

Wireshark is a network packet capture and analysis software ${ }^{[9]}$, it can call WinPCAP components, it can get the network data packets from NIC or virtual network link directly, which can realize the data visualization and network packet flow type detection and analysis in a simple and intuitive way. Through Wireshark, the captured data packet can be directly defined including the filtering conditions, according to the network model, the hierarchical display of different data packet structure can be shown, which is convenient for students to understand the hierarchical structure of the network, network protocol, data structure, network protocol implementation process as well. The most valuable is that through the statistical analysis of the captured packets, various network performance indicators can be displayed in different charts. Therefore, the introduction of Wireshark packet capturing analysis technology to the classroom can not only help beginners understand the concept of network hierarchy directly, but also can help the senior students to analyze and design the network. 


\section{Transmission Control Debugging Tools}

SocketTool is a network transport layer protocol debugging tool, which can simulate the function of testing TCP server, TCP client, UDP server, UDP server, UDP broadcast and multicast and so on. Using SocketTool tools, the network state of TCP connection, TCP port, UDP port as well as the state of service can be visualized, with the analysis of Wireshark protocol, the transmission layer protocol can greatly enhance the work process, especially the establishment of TCP connection. The visualization of sliding window mechanism and releasing connection process of display ability can improve the dynamic state of course information vividly.

\section{Ways of Having Computer Network Course}

Computer Network course is the basic concept of computer network, computer network architecture, physical layer, data link layer, medium access control layer, network layer, transport layer, application layer, network security technology and so on, namely, nine knowledge units, which can be divided into the concept of common sense knowledge, technical knowledge, operation skill principle knowledge and ideas and methods of teachers' summary of the comprehensive knowledge. During the period of having teaching design, according to the different points of knowledge classification, it can design different knowledge expression modes $^{[10][11]}$, so as to complete the teaching preparation. The concept of common sense knowledge is mainly used to describe the fact that "what is", which can also be known as fact knowledge (Know-what). This kind of knowledge mainly uses static / dynamic mind map courseware to present, thus facts and characteristics of association can be presented; technology principle knowledge is mainly used to explain "why" reason, it emphasizes the evolution of dynamic "life cycle", which is also known as the principle of knowledge (Know-why). This kind of knowledge mainly adopts case network dynamic demonstration, SocketTool and Wireshark dynamic analysis and interactive operation can display the "operating state" or "the result of stage"; operation skill knowledge is mainly used for the steps and methods of demonstrating "how to do", which can also be known as knowledge skills (Know-how) ${ }^{[12]}$. This kind of knowledge mainly uses the interactive operation of the virtual network environment (GNS3+VirtualBox+Wireshark) to display the "technical method" and "operation process". During the period of teaching, we can select the corresponding ways of knowledge and expression to complete the knowledge explanation, demonstration, interactive operation as well as exchanging discussion, etc..

\section{Teaching Examples of Computer Network}

\section{Application of Mind Mapping Courseware}

As for concept knowledge, reasonable division of the scope of knowledge should be according to the development of knowledge and evolution of knowledge stratification, so as to establish the link between knowledge points and form a Computer Network knowledge map. Using NovaMind to design mind mapping courseware, namely, the concept knowledge, common knowledge, knowledge of principles, as well as the comprehensive knowledge summarized by teachers presented to students directly, visually in a related way. During the period of classroom teaching, by clicking the knowledge points "+" or "-", in the form of animation to expand or contract the following knowledge points, while switching the display 
of the scope of knowledge, which can keep the switching process in knowledge consistency, without losing the context logic relationship between the knowledge points.

\section{Dynamic Network Teaching Case}

According to the needs of classroom teaching and experimental teaching, by means of GNS3 virtual network environment, it can design Lan sharing network, exchanging Lan network, IP subnet division and Internet, network address translation, network routing and routing table, routing convergence process, IP multi-casting network, IP packets transmission and ARP process, the principle of DHCP network, as well as other dynamic teaching processes. At the same time, WinXP client, Windwos2003 and Linux server can be established based on VirtualBox, and standard network services such as DNS, FTP and WWW can be installed to connect the network services in the teaching case. Fig. 1 is the Topological diagram of teaching case for IP packet transferring and ARP analysis process.

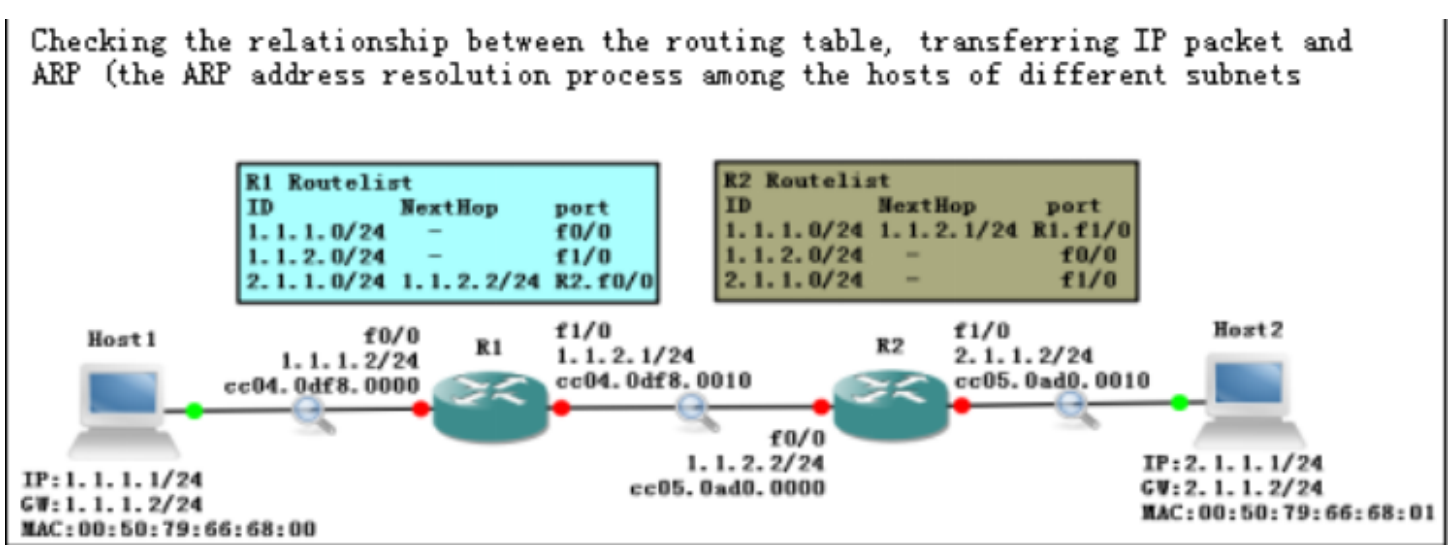

Fig.1 Topological Diagram of Teaching Case for IP Packet Transferring and ARP Analysis Process

\section{Typical Network Cases for Application}

The typical network case for application (Fig.2) is designed to answer the question of the status and function of the relevant knowledge in the course of teaching. The case includes a complicated virtual machine hub, two-layer switcher, router, three-layer switcher, firewall, VPCS simple virtual machine, as well as the complicated virtual machines that can run the client program and web service and so on, which can be connected by fast Ethernet technology to build a shared LAN, exchanging LAN, as well as the corresponding LAN through the router or the three-layer switcher, so as to form the typical Internet architecture of the park. Through VirtualBox virtual machine, the standard network services such as DNS, FTP, Web can be built up; through logical network design, subnet division, IP address division, port IP address configuration, routing configuration technology and some other technologies to achieve the inter-operation ability of campus network. 




Fig. 2 Topology of Cases with

General Campus Network

In order to simulate and display the network scope of DHCP protocol, working process as well as multi-subnet IP address automatic allocation principle, solving the problems that occurred in the teaching material of "DHCP server" and "DHCP scope" question, this case allocated DHCP-Server in router R2-3745 specifically, which can also provide the host computer in each sub-network with dynamic IP address assignment. In order to find out DHCP-Server, subnet gateway interface to the default configuration for the DHCP-Agent in campus network successfully, it can discover data packets forwarded to the DHCP-Server or a DHCP-Agent timely, when host computer can find out DHCP-Server. On this basis, it can complete the simulation of the entrance, firewall protection, DMZ network, network, network teaching experiment and student dormitory network in a typical network in campus network,etc..through the firewall or boundary router, which is connected to ISP network.

\section{Examples of Application of Dynamic Visualization Teaching}

One of the teaching application is when it comes to subnet division, interconnection between routing and network as well as knowledge of routing convergence and so on, the timely introduction of the corresponding teaching cases through the network, by running the operation, the dynamic interactive configuration, checking the network parameters, the routing table router and routing type, routing entries and so on, it can clearly observe the different configuration states before and after the routing aggregation in network.

Another of the teaching application is when it is introducing the 6.8 section "ARP", it can use the network of Fig.1, then firstly cleared the needed routing information, IP address and MAC address information, which is required by "the transferring of IP packet from Host1 to Host2" and "ARP according to the routing segment". Then it can generate IP data stream from Host2 visiting Host1. Finally, using Wireshark to capture and analyze ICMP data packet, so as to observe the Ethernet frame and IP data packet, the description of he Internet transmitting IP packet can show the principle of "IP packet source and destination address is not changed" and "MAC address in piece analytical subsection" simple, which can reflect the relationship between routing table and Internet ARP section analysis in intuitive and accurate way.

The third teaching application is introducing the 8.7 section, when it comes to "DHCP protocol, namely, the host configuration and dynamic host configuration", according to the actual case of subnets in the network, by means of the dynamic visualization approach to 
explain the basic configuration of DHCP-Server content, which can cover the definition of different subnet address pool, as well as the definition of the default network, at the same time, by means of visualization to explain and demonstrate the position, function and configuration of DHCP-Agent, through the experimental method to verify that "DHCP-Agent cannot exceed three grade"of the technical limitations. With Wireshark to capture and analyze DHCP packet, it can make students quickly clear the scope of use of DHCP protocol, the interaction process of protocol, which can completely reveal the problem a "UDP familiar port is without host computer IP address" and "why DHCP protocol can use two UDP familiar ports".

\section{Conclusion}

The introduction of mind mapping courseware can strengthen the visual expression of logic common knowledge in Computer Network. Moreover, it can enhance the ability of summarizing teaching courseware of teaching content, which is easy fir students to develop associative thinking; the proper application of virtual network environment and dynamic network teaching case can transfer the abstract and complicated messy the principle of knowledge into the simple, specific, vivid, practical and operating, network object with "life cycle", through a variety of interactive and visualization tools, in the form of graph to show the network configuration information, operation state and control principle dynamically and intuitively. Plus the teacher's lectures and analysis, it can help students quickly understand the single point of knowledge, so as to establish a "knowledge chain" of the course with connection, regularity and application. The teaching practice showed us that the dynamic visual teaching method enhanced the dynamic content of classroom teaching and students' intuition. It is easier for students to understand, which is also easy to find out problems, discuss, so as to stimulate the students' interests in learning, improving classroom atmosphere as well as teaching effect.

\section{Reference}

[1] Fu Beibei. Network Technology _ Curriculum Design and Implementation of Visual Teaching Resource[D], Shandong: Liao Cheng University, 2016.

[2] Wu Gongyi. Computer Network (The Third Edition)[M]. Beijing: Tsinghua University Press, 2013: 255-258.

[3] Wu Gongyi. Computer Network (The Third Edition)[M]. Beijing: Tsinghua University Press, 2013: 353-356.

[4] Sui Feiyang, etc.. Construction and Application of Portable Network Experiment Environment[J]. Experimental Science and Technology, 2014,02:12-15.

[5] HUAWEI, eNSP Manual[EB/OL]. http://down.51cto.com/data/6431602014.

[6] GNS3, GNS3 Documentation[EB/OL]. https:/www.gns3.com/support/docs/ lossary-of-terms, 2016.

[7] Oracle, the VirtualBox User Manual[EB/OL]. https://www.virtualbox.org/manual/ serManual.html, 2016.

[8] Qemu Documentation/Networking[EB/OL]. http://wiki.qemu.org/Documentation/ network-ing\#Network_backend_types, 2012-12-16. 
[9] Wireshark User’s Guide[EB/OL]. https://www.wireshark.org/docs/ sug_html_chunked/, 2017-05-20.

[10] Zhu Yonghai. Research on Visual Representation Based on Knowledge Classification[D]. Jiangsu: Nanjing Normal University, 2013.

[11] Zhao Huichen. Design Method of Visual Representation of Knowledge Visualization[J]. Open Education Research, 2012, 05: 25-30.

[12] Economy Based ob Knowledge--Organization of Economic Cooperation and Development, 1996 Annual Report[J]. China Business Management Research, 1998, 07: 59-63. 